\title{
A single-chip telescope for heavy-ion identification
}

\author{
G. Pasquali ${ }^{1,2}$,a , S. Barlini ${ }^{1,2}$, L. Bardelli ${ }^{1,2}$, S. Carboni $^{1,2}$, N. Le Neindre ${ }^{3}$, M. Bini ${ }^{1,2}$, B. Borderie ${ }^{4}$, R. Bougault $^{3}$, \\ G. Casini ${ }^{2}$, P. Edelbruck ${ }^{4}$, A. Olmi ${ }^{2}$, G. Poggi ${ }^{1,2}$, M.F. Rivet ${ }^{4}$, A.A. Stefanini ${ }^{1,2}$, G. Baiocco ${ }^{3,5}$, R. Berjillos ${ }^{10}$, \\ E. Bonnet ${ }^{6}$, M. Bruno ${ }^{5}$, A. Chbihi ${ }^{6}$, I. Cruceru ${ }^{14}$, M. Degerlier ${ }^{8}$, J.A. Dueñas ${ }^{10}$, M. Falorsi ${ }^{1}$, E. Galichet ${ }^{4,13}$, \\ F. Gramegna ${ }^{8}$, A. Kordyasz ${ }^{11}$, T. Kozik ${ }^{12}$, V.L. Kravchuk ${ }^{8}$, O. Lopez ${ }^{3}$, T. Marchi ${ }^{8}$, I. Martel ${ }^{10}$, L. Morelli ${ }^{5}$, \\ M. Parlog ${ }^{3,14}$, H. Petrascu ${ }^{14}$, S. Piantelli ${ }^{2}$, E. Rosato ${ }^{7}$, V. Seredov ${ }^{4}$, E. Vient ${ }^{3}$, and M. Vigilante ${ }^{7}$ \\ For the FAZIA Collaboration \\ 1 Dipartimento di Fisica, Università di Firenze, via G.Sansone 1, 50019 Sesto Fiorentino (FI), Italy \\ 2 INFN, Sezione di Firenze, via G.Sansone 1, 50019 Sesto Fiorentino (FI), Italy \\ 3 LPC, IN1P3-CNRS, ENSICAEN et Université de Caen, F-14050 Caen-Cedex, France \\ 4 Institut de Physique Nucléaire, CNRS/IN2P3, Université Paris-Sud 11, F-91406 Orsay cedex, France \\ ${ }^{5}$ INFN and University of Bologna, 40126 Bologna, Italy \\ 6 GANIL, CEA/DSM-CNRS/IN2P3, B.P. 5027, F-14076 Caen cedex, France \\ 7 Dipartimento di Scienze Fisiche e Sezione INFN, Università di Napoli Federico II, I-80126 Napoli, Italy \\ 8 INFN-LNL Legnaro, viale dell'Università 2, 35020 Legnaro (Padova), Italy \\ 9 INFN-LNS Catania, 95129 Catania, Italy \\ 10 Departamento de Fisica Aplicada, FCCEE Universidad de Huelva, 21071 Huelva, Spain \\ 11 Heavy Ion Laboratory, University of Warsaw, ul. Pasteura 5a, 02-093 Warsaw, Poland \\ 12 Jagiellonian University, Institute of Nuclear Physics IFJ-PAN, PL-31342, Kraków, Poland \\ 13 Conservatoire National des Arts et Métiers, 75141 Paris Cedex 03, France \\ 14 "Horia Hulubei" National Institute of Physics and Nuclear Engineering, RO-077125 Bucharest, Romania
}

Received: 26 July 2012 / Revised: 11 September 2012

Published online: 23 November 2012

(C) The Author(s) 2012. This article is published with open access at Springerlink.com Communicated by N. Alamanos

\begin{abstract}
A $\Delta E-E$ telescope exploiting a single silicon chip for both $\Delta E$ measurement and scintillation light collection has been tested. It is a $\mathrm{Si}-\mathrm{CsI}(\mathrm{Tl})$ telescope tailored for mass identification of light charged particles and intermediate mass fragments. A procedure based on two different shaping filters allows for extraction of the $\Delta E-E$ information from the single silicon signal. The quality of the obtained fragment identification is expressed in terms of a figure of merit and compared to that of a standard $\Delta E-E$ telescope. The presented configuration could be a good candidate for the basic cell of a large solid angle array of $\Delta E-E$ telescopes, given the reduction in complexity and cost of the front-end electronics.
\end{abstract}

\section{Introduction}

The effort towards complete characterization of chargedparticle emission in heavy-ion collisions has motivated, in the past decades, the construction of detection arrays covering almost $4 \pi$ in solid angle [1-4].

In order to identify, both in charge and mass, the emitted nuclear fragments, detector arrays usually feature a $\Delta E-E$ telescope as elemental cell. A $\Delta E-E$ telescope is a multi-detector system: the impinging particle traverses the detectors one after the other and the energy deposited in each detector is measured [5]. In some cases two stages are enough and the correlation between the energy $\Delta E$ lost in

\footnotetext{
a e-mail: pasquali@fi.infn.it
}

the first stage and the residual energy $E_{\text {RES }}$ deposited in the second stage allows to identify the charge (and also the mass) of nuclear fragments. However, neither fragments stopped in the first $\Delta E$ detector nor fragments punching through the whole telescope can be uniquely identified. Therefore an overall increased thickness is needed, consisting of three detector stages, e.g., a gas detector, a silicon detector and a scintillator $[1,3]$, to obtain identification in a wider dynamic range. A $\mathrm{CsI}(\mathrm{Tl})$ scintillator is often employed as residual energy detector, due to its relatively low cost, high stopping capability and reasonable energy resolution $[6,7]$.

Mass and charge identification of light charged particles (LCP) and intermediate mass fragments (IMF) will be particularly useful at Radioactive Ion Beam facilities 
in view of studies focused on nuclear isospin phenomena, where the $N / Z$ ratio of the products will be a key experimental observable [8-11].

A new telescope array is under construction by the FAZIA Collaboration [12]. The array is based on threestage $\Delta E-E$ telescopes (Si-Si-CsI $(\mathrm{Tl})$ ). The excellent energy resolution of silicon detectors is exploited to identify heavy fragments stopped in the second element via the $\Delta E-E$ technique. A key feature of FAZIA is the Pulse Shape Analysis (PSA) of detector signals. PSA is employed to identify fragments stopped in the first silicon [13-15], thus lowering the identification thresholds with respect to the standard $\Delta E-E$ technique, while at the same time avoiding the technical complications associated with gas detectors. The second silicon detector (or the combination of the two silicon detectors) and the $\mathrm{CsI}(\mathrm{Tl})$ constitute a $\Delta E-E$ telescope for identifing the most energetic LCP's and IMF's.

Part of the effort in designing FAZIA was devoted to reducing the complexity and cost of the apparatus. To this aim, one could use the second Si detector both as a $\Delta E$ detector and as photodiode for reading out of the $\mathrm{CsI}(\mathrm{Tl})$ scintillation light. This solution, named "SingleChip Telescope" (SCT), was first proposed and tested twenty years ago $[16,17]$ (see [18] for a recent implementation). In ref. [16] the SCT signals were treated with analog electronics and peak-sensing ADC's and the identification capabilities were studied for $\mathrm{H}$ and $\mathrm{He}$ isotopes only. In this work SCT signals have been digitized, using sampling boards developed within the FAZIA Collaboration, and stored for offline analysis. Moreover the SCT identification capability has been studied both for LCP's and IMF's.

When applied to an array covering a large solid angle, the main advantage of the SCT configuration is the reduced number of front-end electronics (FEE) channels with respect to a standard $\Delta E-E$ telescope (a factor of two for a Si-CsI(Tl) telescope), allowing for less crowded frontend, lower power dissipation in vacuum and reduction in cost. As a disadvantage, a dedicated and somewhat critical signal-analysis procedure is needed to extract from a unique signal the information needed for charge and mass identification.

In sect. 2 the experimental setup employed in the SCT beam tests is illustrated. A method for extracting the $\Delta E$ and $E_{\mathrm{RES}}$ information from SCT signals, based on two different shaping filters, is discussed in sect. 3. Another method, based on a fit procedure, will be presented in a forthcoming paper [19]. Section 4 discusses the performance obtained with the "shaper" method and compares it with a standard telescope in which the scintillation light is read out by a dedicated photodiode.

\section{Experimental setup}

The data presented in this work were collected in Catania at Laboratori Nazionali del Sud (LNS) of the INFN. The beam was ${ }^{129} \mathrm{Xe}$ at $35 \mathrm{~A} \mathrm{MeV}$ impinging on a ${ }^{\text {nat }} \mathrm{Ni}$ target.

The SCT tested in this work is part of a $\mathrm{Si}-\mathrm{Si}-\mathrm{CsI}(\mathrm{Tl})$ telescope (the TeleC of ref. [15]): the SCT constitutes the
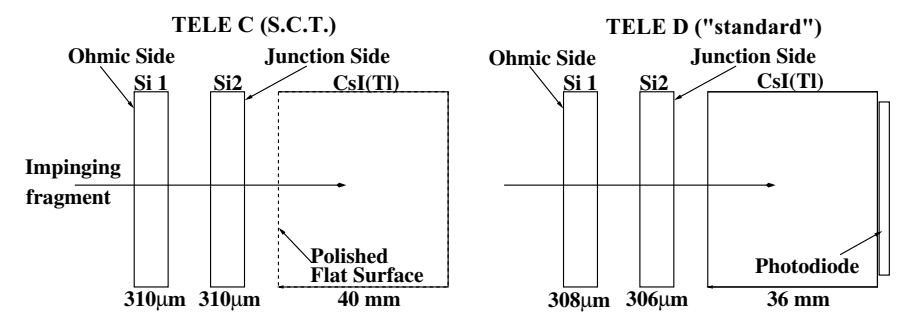

Fig. 1. Schematic drawing of telescope $\mathrm{C}$ and D tested at LNS. In telescope C, the second silicon ( $\mathrm{Si} 2)$ and the $\mathrm{CsI}(\mathrm{Tl})$ are mounted as a SCT configuration.

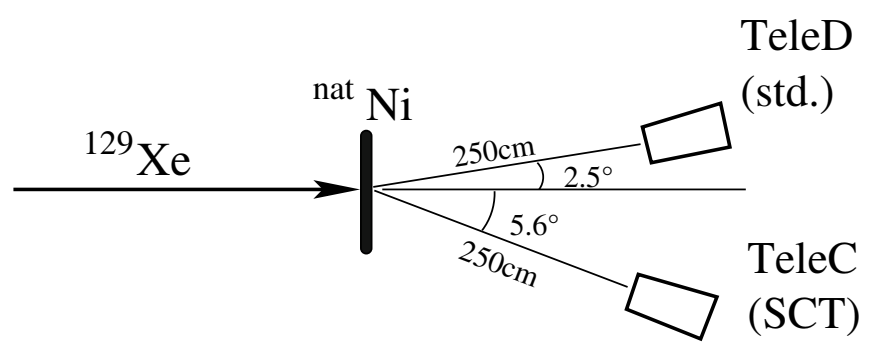

Fig. 2. Position of telescopes $\mathrm{C}(\mathrm{SCT})$ and D (standard $\Delta E$ $E)$ with respect to the beam and target.

second and third stage of the telescope while the first silicon detector acts as a standard $\Delta E$ detector (see fig. 1).

A standard Si-Si-CsI(Tl) telescope ("TeleD") of similar characteristics with respect to the SCT was also present. Both telescopes were optimized for particle identification according to the FAZIA recipe for silicon detectors, described elsewhere [20,21], which allows for excellent results $[14,15]$.

TeleD will be used as a reference in order to compare the SCT concept with the standard $\Delta E-E$ technique in terms of particle identification capability. Both telescopes were placed downstream with respect to the target, on a horizontal plane containing the beam axis, about $250 \mathrm{~cm}$ from the target. The polar angle with respect to the beam was $2.5^{\circ}$ for TeleD and $5.6^{\circ}$ for the SCT (see fig. 2).

In order to get better particle identification from PSA, all silicon detectors were mounted with the ohmic side facing the target so that particles enter the detector from the low field face $[13,14]$. The silicon detectors, with an active area of $20 \mathrm{~mm} \times 20 \mathrm{~mm}$, are made of neutron transmutation doped (nTD) silicon having a resistivity of about $3 \mathrm{k} \Omega \mathrm{cm}$.

Actual detector thicknesses, as measured with a precision micrometer, were found to lie between $306 \mu \mathrm{m}$ and $310 \mu \mathrm{m}$ (see table 1). A key factor for particle identification with the $\Delta E-E$ technique is the thickness uniformity of the $\Delta E$ detector, which according to our measurements is quite good, better than $\pm 1 \mu \mathrm{m}$. A $20 \mathrm{~nm}$ thick aluminum layer was deposited on the ohmic side, while on the junction side only a very thin silicon dioxide layer is present. The junction side is thus sensitive to visible light photons with almost unit quantum efficiency, a mandatory feature for employing the detector as a regular silicon photodiode. 
Table 1. Detector characteristics: telescope type, thicknesses of telescope stages, gain and full scale of the associated FEE, sampling rate and physical number of bits of the digitizers. The full scale energy value takes into account the position of the signal baseline in the ADC range. Gain is given in $\mathrm{keV} / \mathrm{LSB}$ units, where LSB is the Least Significant Bit of the ADC.

\begin{tabular}{|l|l|l|l|}
\hline & & Tele C & Tele D \\
\hline Type & & SCT & Standard $\Delta E-E$ \\
\hline \multirow{5}{*}{ Si1 } & thick. & $310 \mu \mathrm{m}$ & $308 \mu \mathrm{m}$ \\
& Gain & $280 \mathrm{keV} / \mathrm{LSB}$ & $300 \mathrm{keV} / \mathrm{LSB}$ \\
& F.S. & $3.5 \mathrm{GeV}$ & $3.5 \mathrm{GeV}$ \\
& SR & $100 \mathrm{MHz}$ & $100 \mathrm{MHz}$ \\
& bits & 14 & 14 \\
\hline \multirow{5}{*}{ Si2 } & thick. & $310 \mu \mathrm{m}$ & $306 \mu \mathrm{m}$ \\
& Gain & $40 \mathrm{keV} / \mathrm{LSB}$ & $160 \mathrm{keV} / \mathrm{LSB}$ \\
& F.S. & $0.5 \mathrm{GeV}$ & $2.0 \mathrm{GeV}$ \\
& SR & $100 \mathrm{MHz}$ & $100 \mathrm{MHz}$ \\
& bits & 14 & 14 \\
\hline \multirow{5}{*}{ CsI(Tl) } & thick. & $40 \mathrm{~mm}$ & $36 \mathrm{~mm}$ \\
& Gain & & $50 \mathrm{keV}(\mathrm{Si}) / \mathrm{LSB}$ \\
& F.S. & \multirow{2}{*}{ see Si2 } & $0.2 \mathrm{GeV}(\mathrm{Si})$ \\
& SR & & $125 \mathrm{MHz}$ \\
& bits & & 12 \\
\hline
\end{tabular}

A square brass collimator $(20 \mathrm{~mm} \times 20 \mathrm{~mm})$ was mounted in front of both telescopes, to prevent particles from hitting silicon detector borders (i.e. outside the active area).

In the SCT, the $\mathrm{CsI}(\mathrm{Tl})$ dimensions are $20 \mathrm{~mm} \times$ $20 \mathrm{~mm} \times 40 \mathrm{~mm}$; all surfaces are sanded and covered with Millipore paper except for the front face, facing the silicon detector, which has been polished, making it optically transparent: scintillation photons can reach the photosensitive surface of the silicon detector and be collected (see fig. 1).

The TeleD features a $20 \mathrm{~mm} \times 20 \mathrm{~mm} \times 36 \mathrm{~mm} \mathrm{CsI(Tl)}$ scintillator with photodiode read-out. The front face of the $\mathrm{CsI}(\mathrm{Tl})$ is covered with aluminized Mylar, $2 \mu \mathrm{m}$ thick; the sides are sanded and covered with Millipore paper; the rear face has been polished to make it optically transparent and glued to a $18 \mathrm{~mm} \times 18 \mathrm{~mm}$ active area photodiode (by Hamamatsu Photonics).

Each silicon detector is connected to a PACI [22] preamplifier with charge and current outputs, both driven by differential output buffers. Signals from the PACI are brought out of the vacuum chamber to the digitizing boards through $8 \mathrm{~m}$ long differential cables (BELTEN Twinax 9271, characteristic impedance $120 \Omega$ ). Also the photodiode of TeleD is connected to a dedicated PACI preamplifier.

The present FAZIA digitizing boards feature two channels (one for the charge and one for the current output of the PACI). Only charge signals have been analyzed in this work. The analog input stage exploits a 3-pole lowpass anti-aliasing filter (Sallen-Key configuration, Bessel frequency response [23]). The ADC (LTC2254 by Linear Technology [24]) has a $100 \mathrm{MHz}$ sampling rate and 14

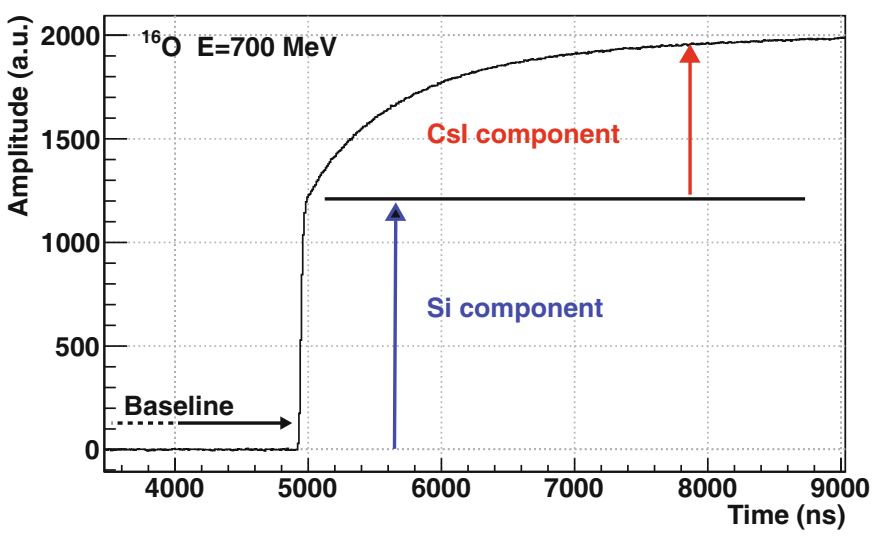

Fig. 3. SCT output signal for a $700 \mathrm{MeV}{ }^{16} \mathrm{O}$ fragment. Polezero cancellation of the decay of the charge preamplifier has been applied.

physical bits. The effective number of bits of the custom board is $\approx 11.2$. In the present experiment, FAZIA boards have been used for the signals of the Si detectors.

The PACI output of the photodiode has been sampled by a digitizing board developed at INFN-Florence [25] featuring a 12 bit ADC with $125 \mathrm{MHz}$ sampling rate. Detector characteristics, sampling rates and bit resolutions are summarized in table 1.

The SCT scintillation light contributes to the signal of the second silicon detector. As an example, a digitized $\mathrm{SCT}$ signal is shown in fig. 3. The signal refers to an ${ }^{16} \mathrm{O}$ of $700 \mathrm{MeV}$ total energy which crossed the Si chip and was stopped in the $\mathrm{CsI}(\mathrm{Tl})$. A "pretrigger" portion, a few $\mu \mathrm{s}$ long, is acquired for every signal: it is used for the offline calculation of the baseline that is needed for signal amplitude estimation [26]. After the baseline portion (ending at about $4.9 \mu \mathrm{s}$ in fig. 3) a steep rise is observed (rise time $\approx 50 \mathrm{~ns}$ ), associated with energy deposited directly in the silicon. The superimposed slower component due to the collection of scintillation light from the $\mathrm{CsI}(\mathrm{Tl})$ can be easily recognized.

\section{Data analysis with "shapers" method}

\section{1 "Shapers" method basic principle}

In the SCT, the charge carriers in the silicon (i.e. electronhole pairs) result either from the direct ionization of particles or from collection of scintillation photons: let us call $Q_{I}$ the charge associated with direct ionization and $Q_{L}$ that associated with the produced photons. Then $Q_{I}$ is proportional to the energy $\Delta E$ deposited by the particle in the silicon while $Q_{L}$ is proportional to the residual energy $E_{\mathrm{RES}}$, i.e. the energy deposited by the particle in the $\operatorname{CsI}(\mathrm{Tl})$ scintillator. Both $Q_{I}$ and $Q_{L}$ contributions are integrated by a single PACI charge preamplifier, thus combining in the same signal the $\Delta E$ and the $E_{\mathrm{RES}}$ information.

It is known that the decay of $\mathrm{CsI}(\mathrm{Tl})$ scintillation light as a function of time can be described by the sum of two exponentials with different time constants: a fast time constant $\tau_{f}$ and a slow time constant $\tau_{s}$. As shown in fig. 3, 
charge collection in the Si detector and light emission in the scintillator have quite different time scales: both $\tau_{f}$ and $\tau_{s}$ are longer than the typical charge collection time in the silicon.

The different time scales make it possible to disentangle $\Delta E$ and $E_{\mathrm{RES}}$. This task can be accomplished, e.g., by using analog shapers with different time constants, as in ref. [16]. A similar treatment can also be implemented on digitized waveforms: the digitized signal is duplicated and the two copies are processed via different digital shapers with different time constants. The data presented in this paper have been processed with semi-Gaussian filters. Using other filters (e.g., trapezoidal shapers), gives practically the same results, provided that the time response of the filters is suitably optimized. In the following, the shaper with the shorter time constant will be called "short" shaper, the other will be called "long" shaper.

Let us call $\mathrm{S}$ and $\mathrm{L}$ the peak amplitudes of the short and long shaped signals, respectively ${ }^{1}$. Possible model estimates of these amplitudes are $\hat{\mathrm{S}}$ and $\hat{\mathrm{L}}$, defined as

$$
\hat{\mathrm{S}}=Q_{I}+\alpha Q_{L} ; \quad \hat{\mathrm{L}}=Q_{I}+\beta Q_{L},
$$

where a unit conversion factor is assumed between collected charge and shaper amplitude. Here we have assumed that the peaking times of the two shapers are much longer than the collection time of the electron-hole pairs in silicon, so that the charge $Q_{I}$ is totally collected. The coefficients $\alpha$ and $\beta$, on the contrary, represent the two different fractions of $Q_{L}$ contributing to $\mathrm{S}$ and $\mathrm{L}$. These fractions depend on the magnitude of the chosen shaping constants with respect to the scintillation time constants $\tau_{f}$ and $\tau_{s}$. For the $\mathrm{CsI}(\mathrm{Tl})$ scintillators employed during the FAZIA tests a good estimate is $\tau_{f} \simeq 750 \mathrm{~ns}$ and $\tau_{s} \simeq 5 \mu \mathrm{s}$.

Solving eqs. (1) for $Q_{I}$ and $Q_{L}$ one gets

$$
Q_{I}=\frac{\beta}{\beta-\alpha}\left(\hat{\mathrm{S}}-\frac{\alpha}{\beta} \hat{\mathrm{L}}\right) ; \quad Q_{L}=\frac{1}{\beta-\alpha}(\hat{\mathrm{L}}-\hat{\mathrm{S}}) .
$$

To simplify the treatment one might consider a short peaking time $T_{S} \ll \tau_{f}$ and a long peaking time $T_{L} \gg \tau_{s}$, a choice which would lead to $\alpha=0$ and $\beta=1$ in eqs. (1) and (2). However this is not practical.

In fact, electronic noise must be taken into account: $T_{S}$ values lower than about $400 \mathrm{~ns}$ produce a too low signalto-noise ratio because of the unavoidable high-frequency noise at the output of the preamplifier. Moreover, $T_{L}$ is limited by the finite length of the digitized signal (about $30 \mu \mathrm{s}$ ) and by the unavoidable low-frequency noise of the preamplifier. So a viable compromise has to be found. In this work, the short shaper has a peaking time $T_{S}=700 \mathrm{~ns}$ and the long shaper has a peaking time $T_{L}=8 \mu \mathrm{s}$. A digital pole-zero cancellation of the exponential decay of the PACI preamplifier is performed on all signals before applying the shaping filters.

\footnotetext{
1 We call the shaper amplitudes S and L (for "short" and "long"), instead of $F$ and $S$ (for "fast" ans "slow") as in ref. [16]. We reserve the terms "fast" and "slow" for the two components of $\mathrm{CsI}(\mathrm{Tl})$ scintillation light.
}

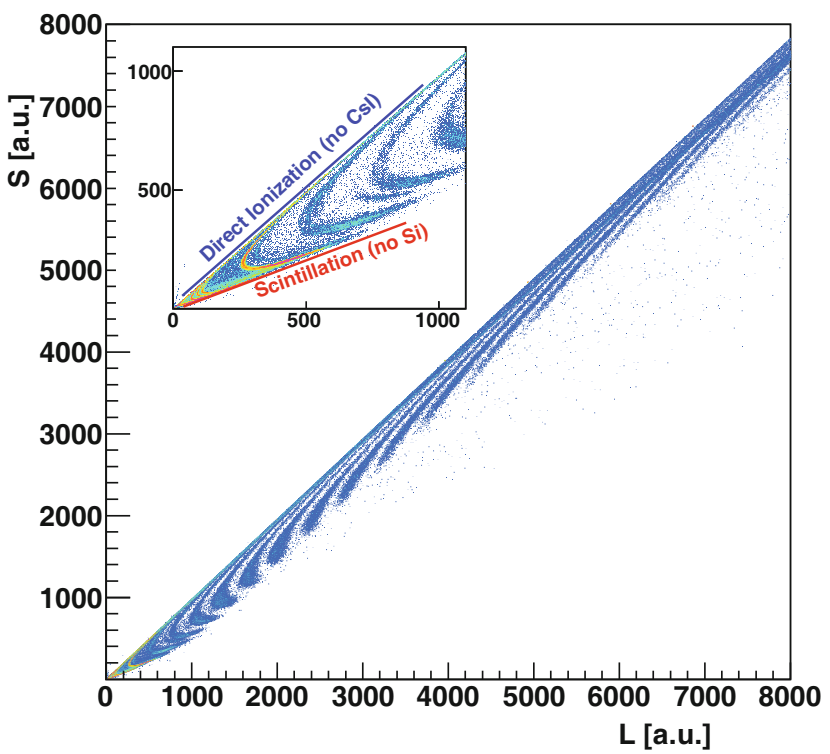

Fig. 4. Short shaper amplitude, S, versus long shaper amplitude, L. The inset shows an expanded view of the low energy part. The direct ionization and the scintillation lines are evidenced: all events must fall between these two lines (see ref. [16]). Counts are plotted on a logarithmic scale.

Figure 4 shows an example of the experimental $\mathrm{S} v s$. L correlation. Events with $Q_{L}=0$ lie along the top border of the $\mathrm{S}$ vs. L correlation ("direct ionization" line in the inset in fig. 4). Events with $Q_{I}=0$ (e.g., cosmics, neutrons, gammas) fall along the bottom border ("scintillation" line). Applying eqs. (2) to the data, we can reconstruct the $\Delta E$ and $E_{\text {RES }}$ information (in arbitrary units) as follows:

$$
\Delta E \propto \mathrm{S}-K_{L} \mathrm{~L}, \quad E_{\mathrm{RES}} \propto \mathrm{L}-K_{S} \mathrm{~S},
$$

where $K_{L}=\alpha / \beta$ and $K_{S}=1$. The $K_{L}$ and $K_{S}$ coefficients can be adjusted, by trials and errors, until the events with $Q_{L}=0$ become parallel to the ordinate axis and those with $Q_{I}=0$ become parallel to the abscissa. As a result of such an adjustment, $\Delta E v s$. $E_{\mathrm{RES}}$ plots like that shown in fig. 5 are obtained (where $K_{L}=0.4$ and $K_{S}=1.02$ ).

In figs. 4 and 5 , events associated with IMF stopped in the silicon are included. Actually, they overlap with the familiar $\Delta E-E$ correlation, as it clearly appears in fig. 5 . The effect is due to the relatively long time needed for collecting the direct ionization of IMF and heavier fragments: the short and the long shaper, having different time constants, actually perform a PSA on Si-stopped ions (the almost vertical lines associated with different $Z$ values can clearly be recognized on the left in fig. 5). These events must first be recognized and excluded from the analysis in order to select proper $\mathrm{Si}-\mathrm{CsI}(\mathrm{Tl})$ events. Their presence was not noticed in ref. [16], probably due the low statistics accumulated in the IMF part of the spectra (see sect. 3.2).

The $K_{S}$ value needed to get the correlation of fig. 5 is greater than the expected unit value. This is due to 


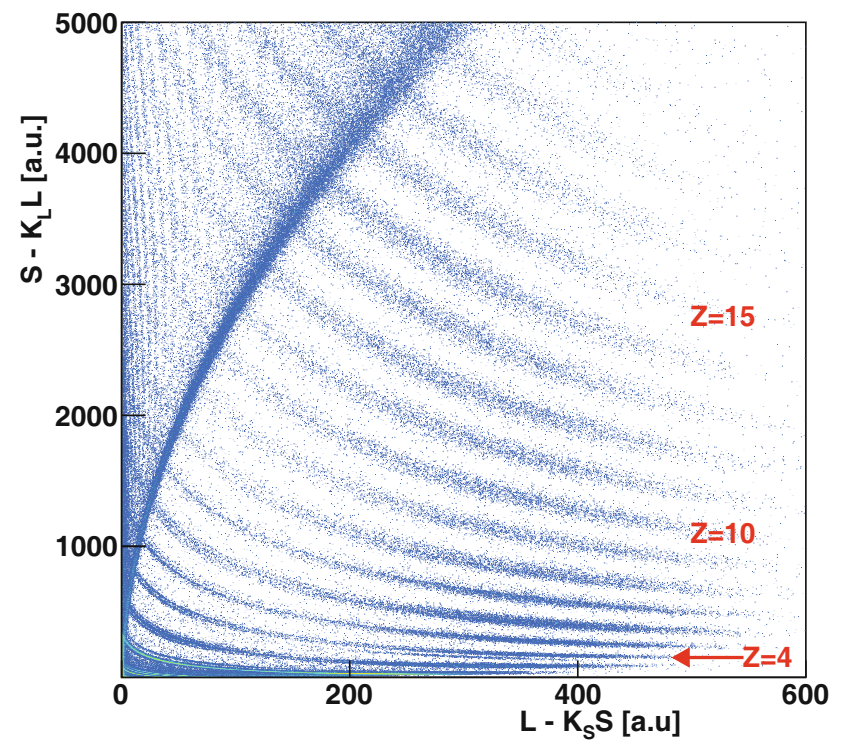

Fig. 5. Reconstructed $\Delta E-E_{\mathrm{RES}}$ plot. The constants $K_{S}$ and $K_{L}$ have been adjusted $\left(K_{S}=1.02, K_{L}=0.4\right)$ to get the proper look of the correlation (see sect. 3.1). The ridge rapidly rising from the origin and the vertical lines in the upper left part are associated with fragments stopped in the silicon. Counts are plotted on a logarithmic scale.

the finite rise time of the direct ionization signal, which suffers some ballistic deficit when processed by the short shaper with respect to the long one [27]. The minimum of this rise time is determined by the FEE. Indeed a ballistic deficit of about $2 \%$ has been estimated for the short shaper by studying its response to signals produced in the first silicon, Si1, which are due to direct ionization only and do not contain any $\mathrm{CsI}(\mathrm{Tl})$ contribution. Therefore, in the first of eqs. (1) the coefficient of $Q_{I}$ should be 0.98 instead of 1 .

\subsection{Recognizing fragments stopped in Si detector}

The shapes of the charge signals are quite different for ions stopped in the Si detector with respect to ions producing scintillation also in the $\mathrm{CsI}(\mathrm{Tl})$. A particle stopped in $\mathrm{Si}$ features a steep almost linear leading edge (as shown in the top panel of fig. 6 for a ${ }^{16} \mathrm{O}$ fragment of $285 \mathrm{MeV}$ kinetic energy). On the contrary, when scintillation light contributes to the pulse shape, the topmost part of the leading edge presents a noticeable slowing down, as shown in the bottom panel of fig. 6. Events can be sorted in two classes ("stopped in Si" and "stopped in $\mathrm{CsI}(\mathrm{Tl})$ ") on the base of their different shapes. For example, one could define a parameter $\Delta t_{\text {sym }}$ as

$$
\Delta t_{\mathrm{sym}}=\left(T_{90 \%}-T_{80 \%}\right)-\left(T_{80 \%}-T_{50 \%}\right),
$$

where $T_{N \%}$ is the time at which the leading edge reaches $N \%$ of the maximum amplitude. The rationale behind the $\Delta t_{\text {sym }}$ parameter is illustrated in fig. 6 where the circles

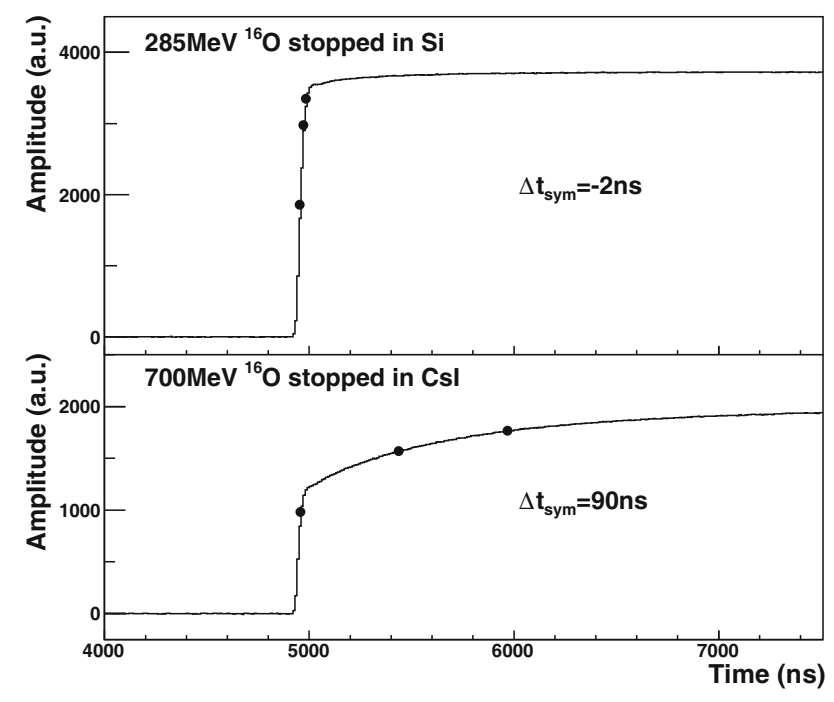

Fig. 6. Typical signal shapes for the two event classes ("stopped in Si" and "stopped in CsI(Tl)"). Top panel: charge signal produced by a ${ }^{16} \mathrm{O}$ with $285 \mathrm{MeV}$ kinetic energy, stopped in the silicon. Bottom panel: charge signal produced by a ${ }^{16} \mathrm{O}$ with $700 \mathrm{MeV}$ kinetic energy, stopped in the CsI(Tl). Full circles plotted on the leading edge correspond to the amplitude fractions of $50 \%, 80 \%$ and $90 \%$, used to calculate $\Delta t_{\mathrm{sym}}$, and to the associated times, namely $T_{50 \%}, T_{80 \%}$ and $T_{90 \%}$ (see sect. 3.2).

mark the signal amplitudes at the adopted values $T_{50 \%}$, $T_{80 \%}$ and $T_{90 \%}$. When the amplitude of the silicon component is greater than $50 \%$ of the full amplitude, the first point (the one at $T_{50 \%}$ ) lies on the steepest part of the leading edge. If there is no $\mathrm{CsI}(\mathrm{Tl})$ contribution to the signal, the other two points will also lie on the steep rise, giving low $\Delta t_{\text {sym }}$ values $\left(e . g ., \Delta t_{\text {sym }}=0\right.$ for an exactly linear leading edge and equally distanced fractions). If the signal slows down in the upper part, as for particles reaching the $\mathrm{CsI}(\mathrm{Tl}), \Delta t_{\text {sym }}$ will tend to become positive. With the chosen fractions, particles stopped in the silicon give $\Delta t_{\text {sym }}<\Delta t_{0}$ while particles stopped in the $\mathrm{CsI}(\mathrm{Tl})$ give values $\Delta t_{\text {sym }}>\Delta t_{0}$, with $\Delta t_{0} \approx 4.1 \mathrm{~ns}$.

When the silicon contribution amounts to less than $50 \%$ of the final amplitude, $\Delta t_{\text {sym }}$ alone does not allow a clean separation. For those events, however, a simple criterium based on signal rise time $(10 \%$ to $90 \%$ of maximum value) can be adopted, since the slow $\mathrm{CsI}(\mathrm{Tl})$ component dominates the leading edge shape. To summarize, events are recognized as

$$
\begin{aligned}
& \text { - "stopped in } \mathrm{CsI}(\mathrm{Tl}) \text { " if }\left(\Delta t_{\mathrm{sym}}>\Delta t_{0}\right) \text { or (signal rise } \\
& \quad \text { time }>600 \mathrm{~ns}) ; \\
& \text { - "stopped in } \mathrm{Si} \text { " if }\left(\Delta t_{\mathrm{sym}}<\Delta t_{0}\right) \text { and (signal rise time } \\
& \quad<600 \mathrm{~ns}) \text {. }
\end{aligned}
$$

Data on charge and mass identification of "stopped in Si" fragments will not be presented in this paper. However, the PSA techniques already discussed in ref. [15] have also been applied to "stopped in Si" events, obtaining results comparable to those of ref. [15]. 


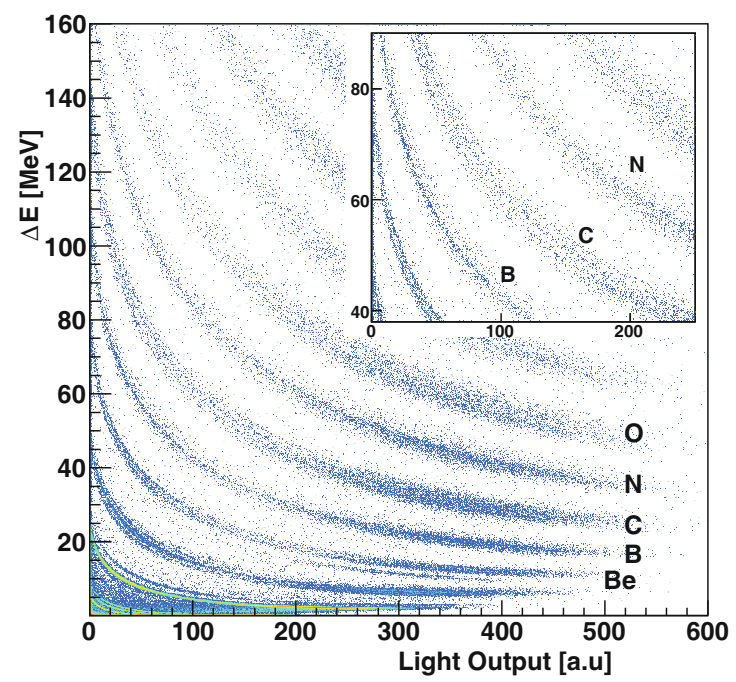

Fig. 7. Reconstructed $\Delta E$ (in $\mathrm{MeV}$ ) vs. $\mathrm{CsI}(\mathrm{Tl})$ light output (a.u.) for IMFs. In the inset, an expanded view of the region where carbon isotopes are better resolved is shown. Counts are plotted on a logarithmic scale.

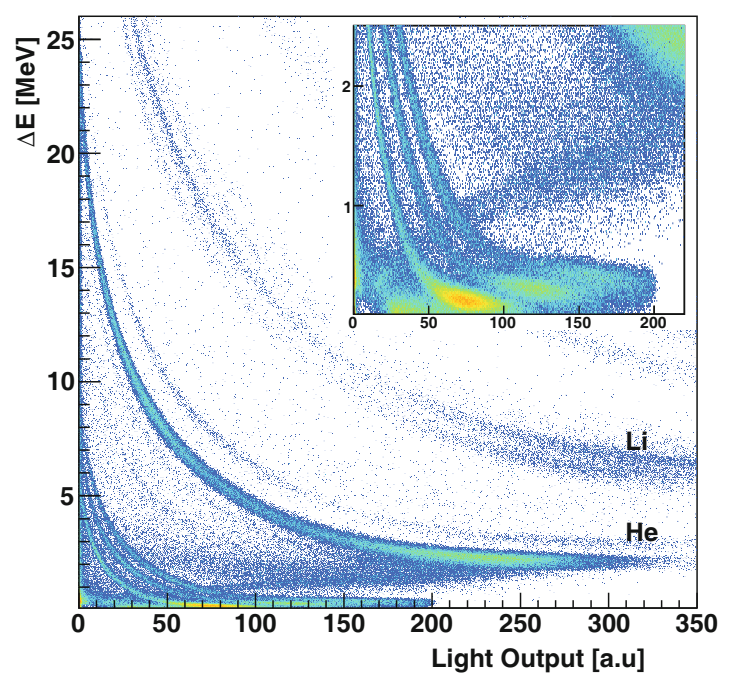

Fig. 8. Reconstructed $\Delta E$ (in $\mathrm{MeV}$ ) vs. $\mathrm{CsI}(\mathrm{Tl})$ light output (a.u.) for LCPs. In the inset, an expanded view of highest energy hydrogen isotopes is shown. Counts are plotted on a logarithmic scale.

\section{3 "Shaper" method results}

Using the selection criteria of sect. $3.2^{2}$, one can produce the "cleaned up" $\Delta E-E_{\mathrm{RES}}$ correlations shown in figs. 7 and 8 where $\Delta E$ and $E_{\mathrm{RES}}$ are calculated as in fig. 5 . Knowing the detector thickness, an energy calibration can be obtained exploiting the punch through points in the

\footnotetext{
${ }^{2}$ A residual contamination of the $\Delta E-E_{\mathrm{RES}}$ correlations by stopped particles remains for hydrogen and helium isotopes. However, since these events fall on the $\Delta E$ axis in the $\Delta E$ $E$ correlation, they can be easily discarded. In fact, the fast collection time for the charge produced by a LCP makes $\mathrm{S}=\mathrm{L}$ and therefore $Q_{L}=0$, see eqs. (2).
}

uncalibrated $\Delta E\left(\mathrm{Si}_{1}\right)$ vs. $\Delta E\left(\mathrm{Si}_{\mathrm{SCT}}\right)$ correlation and the corresponding energy values obtained from energy loss tables [28]. The $\Delta E$ value in figs. 7 and 8 has been calibrated in energy in this way. A possible cause of non-linearity in the $\Delta E$ energy calibration, related to the response of the shapers to the SCT signal, has been identified: it is discussed in the appendix.

The maximum energy deposited in the $\mathrm{CsI}(\mathrm{Tl})$ was $\approx 100 \mathrm{MeV}$ for $\mathrm{H}, \approx 300 \mathrm{MeV}$ for $\mathrm{Li}$ and $\mathrm{Be}, \approx 400 \mathrm{MeV}$ for B and C. It would be possible to calibrate in energy the "Light Output" axis - exploiting the calibrated $\Delta E\left(\mathrm{Si}_{1}\right)$ and $\Delta E\left(\mathrm{Si}_{\mathrm{SCT}}\right)$ values - to obtain full calibrated energy spectra. This has not been attempted in this paper, but we plan to perform the relevant energy calibration when the detector is used in real experiments.

Element resolution is obtained in the whole range allowed by the FEE saturation amplitude (up to $Z \approx 13$ ). A reasonable mass separation is achieved for LCP's and low- $Z$ IMF's (up to Be). However, only marginal mass resolution is achieved for boron and carbon isotopes: different masses can be recognized only in a small energy range. No mass resolution is achieved for $Z>6$.

The $\Delta E-E_{\mathrm{RES}}$ correlations have been subsequently linearized, extracting a particle identification (PID) parameter to quantitatively estimate the isotopic resolution. Results of this analysis will be shown in sect. 4.1 and compared with a similar analysis performed on TeleD.

\section{Detector performance}

\subsection{Particle identification}

Particle identification capabilities can be quantitatively estimated by linearizing the correlations of fig. 7 and fig. 8 in order to extract a particle identification parameter PID. For a comparison of the SCT performance with that of a standard $\Delta E-E$ telescope of similar characteristics, the corresponding $\Delta E-E$ correlations have been obtained for TeleD.

Figure 9 shows the PID spectra for isotopes of elements up to carbon. Comparing the TeleD data (solid black line) and the SCT data (dotted red line) one can notice a comparable performance for LCP's, while for $Z>2$ the SCT identification is significantly worse.

The information given in fig. 9 can be made more quantitative by estimating a "figure of merit" (FoM). For a pair of neighboring peaks, the FoM is defined [29] as

$$
\mathrm{FoM}=\frac{\left|C_{1}-C_{2}\right|}{\mathrm{FWHM}_{1}+\mathrm{FWHM}_{2}},
$$

where $C_{1}$ and $C_{2}$ are the centroids of the two peaks and $\mathrm{FWHM}_{1}$ and $\mathrm{FWHM}_{2}$ are the corresponding full widths at half maximum. Centroids and full widths at half maximum have been estimated from a multiple Gaussian fit. We conventionally consider two isotopes as well separated if $\mathrm{FoM} \geq 0.7[14,15]$.

In order to check how the particle identification degrades with energy, in fig. 10 the FoM for different isotope 

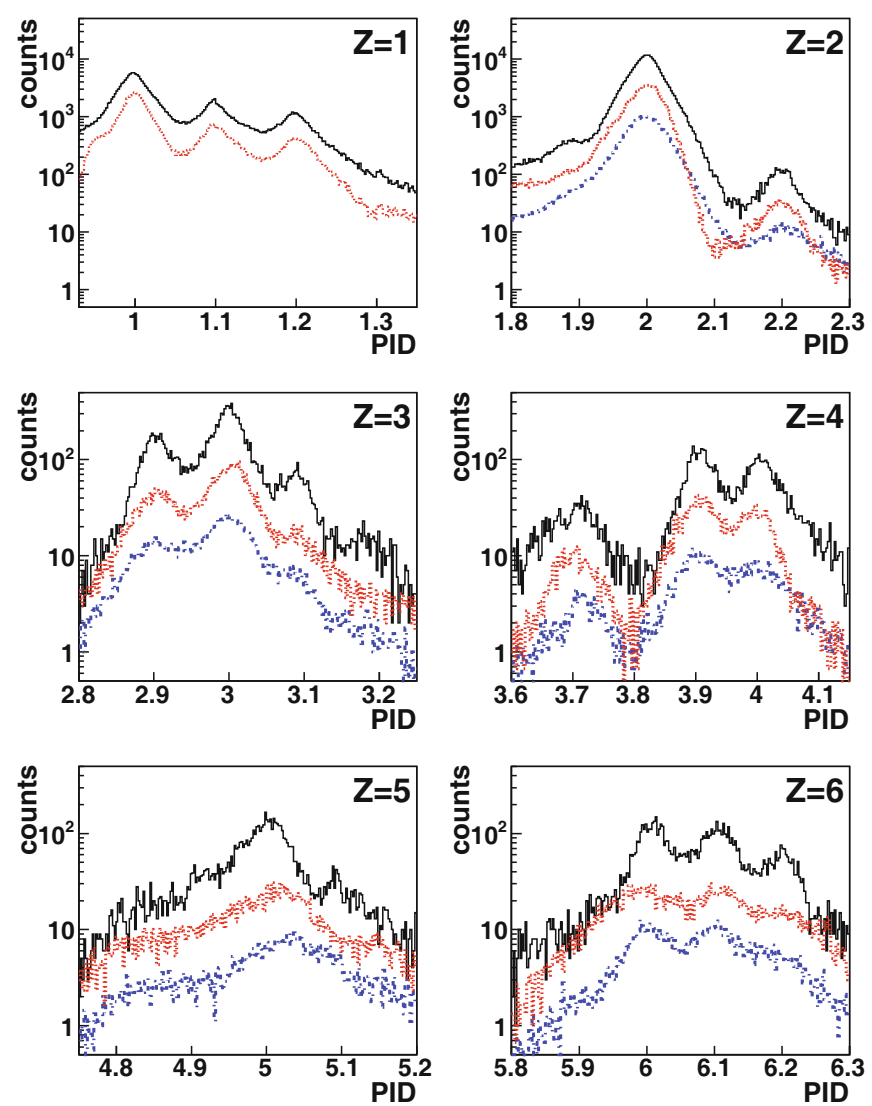

Fig. 9. Particle identification (PID) parameter obtained from the $\Delta E-E$ correlations of figs. 7 and 8: no cut on particle energy has been applied. Solid (black) line: PID spectra from the standard telescope (TeleD). Dotted (red) line: PID spectra from the SCT, downscaled by a factor of three. Dash-dotted (blue) line: PID spectra obtained by mixing together the $\Delta E$ and $E_{\mathrm{RES}}$ signals of TeleD to mimic a SCT (see sect. 4.2), downscaled by a factor of nine.

pairs is plotted as a function of the estimated particle energy, $E_{\mathrm{INC}}$, at the entrance of the silicon of the SCT. To do so, first PID spectra have been obtained for adjacent intervals of light output of the $\mathrm{CsI}(\mathrm{Tl})$ and the FoM values of various isotope pairs have been calculated in each slice. Then for each slice and isotope pair (i.e. for each point in fig. 10) the average $E_{\mathrm{INC}}$ value of the lower-mass isotope has been estimated from the $\Delta E$ measured by Si1 and from energy loss tables [28].

As already noticed in fig. 9, the FoM values for LCP's confirm that a comparable performance can be obtained from the standard telescope (full circles) and the SCT (triangles). For IMF's, on the contrary, the SCT is not as good as the standard telescope, especially at low energies.

\subsection{Reconstruction procedure and isotopic identification}

The worse isotopic resolution of the SCT could be due just to the reconstruction procedure itself. In order to eval-
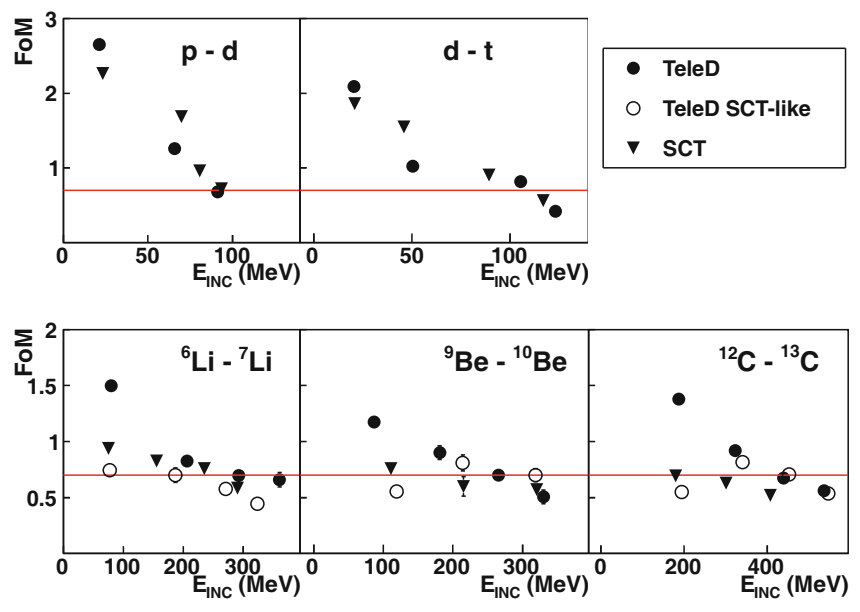

Fig. 10. Figure of Merit (FoM) values for different isotope pairs as a function of the average incident energy $E_{\mathrm{INC}}$ of the first isotope ( $\mathrm{p}, \mathrm{d},{ }^{6} \mathrm{Li},{ }^{9} \mathrm{Be},{ }^{12} \mathrm{C}$, respectively) before entering the SCT. The results are for the SCT (triangles) and for a standard telescope (TeleD). For the latter, Si and $\mathrm{CsI}(\mathrm{Tl})$ signals have been treated separately (full dots) or mixed in a single composite signal (open dots), see sect. 4.2. The incident energy is calculated on the basis of the $\Delta E$ energy measured by the preceding first silicon. Each point corresponds to an interval of $\mathrm{CsI}(\mathrm{Tl})$ Light Output values (see sect. 4.1 for details). The 0.7 limit is evidenced by the horizontal (red) line. Error bars refer only to statistical uncertainties.

uate the effect of the SCT reconstruction procedure on isotopic identification, we have built "SCT-like" signals from the $\mathrm{Si} 2$ and $\mathrm{CsI}(\mathrm{Tl})$ signals of TeleD. The two signals have been first properly scaled in amplitude to take into account the different gains of the associated electronics. Then their leading edges have been time-aligned. The $\mathrm{CsI}(\mathrm{Tl})$ signal sampling period (originally $8 \mathrm{~ns}$ ) has been made equal to that of the Si signal $(10 \mathrm{~ns})$ via interpolation. Finally the two signals have been added sample by sample. The resulting signal looks very much like the proper SCT signal already presented in figs. 3 and 6 . The ADC noise prevents a proper alignment of $\mathrm{Si} 2$ and $\mathrm{CsI}(\mathrm{Tl})$ signals of TeleD for hydrogen isotopes, especially at the highest measured energies: therefore we will not present results for $Z=1$ isotopes.

Signals obtained in such a way have been analyzed using the same reconstruction procedure as for the actual SCT signals. $\Delta E-E_{\mathrm{RES}}$ correlations similar to those of figs. 7 and 8 have been produced and linearized. It is thus possible to obtain the PID spectra shown by the dash-dotted (blue) histograms in fig. 9. A worsening of the particle identification capability due to the reconstruction procedure is apparent when comparing PID obtained with the standard treatment (solid black line) and with the SCT-like analysis (dash-dotted blue line).

To be more quantitative, the FoM for neighboring isotopes is plotted in fig. 10 (open circles). Comparing the FoM values obtained for TeleD with the two methods (full and open circles in fig. 10), we find that the reconstruction procedure worsens the resolution particularly at low 
energy. One could then attribute the worse FoM values obtained at the lowest energies for the SCT, with respect to the TeleD standard, to the need to extract the information from a single signal. It is well known that the isotopic resolution is influenced more by the resolution in $\Delta E$ than in $E_{\mathrm{RES}}$. In a $\mathrm{Si}-\mathrm{CsI}(\mathrm{Tl})$ telescope, the $E_{\mathrm{RES}}$ measurement is certainly more affected by statistical fluctuations than the $\Delta E$ measurement, due to the lower number of carriers. In fact, taking into account light collection efficiency, scintillation photons in $\mathrm{CsI}(\mathrm{Tl})$ have an energy cost at least a factor of 20 larger than electron-hole pairs in Si. When $\Delta E$ is obtained from a single signal combining the $\Delta E$ and $E_{\mathrm{RES}}$ information, the larger fluctuations in $E_{\mathrm{RES}}$ can negatively affect the reconstructed $\Delta E$ value, thus spoiling the isotopic resolution with respect to a standard telescope where $\Delta E$ and $E_{\mathrm{RES}}$ are treated separately.

In the PID spectra of fig. 9, TeleD and SCT present similar isotopic separation for hydrogen isotopes even at the lowest energy. In fact, as shown in fig. 10, at the lowest energy the SCT has a better isotopic separation for hydrogen isotopes than for IMF's. A possible interpretation of this result is the following: the FEE electronics of the SCT features a better $S / N$ ratio than TeleD, due to the higher gain of the associated PACI preamplifier. In fact, the energy range allowed by the FEE was about $500 \mathrm{MeV}$ for the SCT and about $2 \mathrm{GeV}$ for the $\mathrm{Si} 2$ of TeleD. For LCP's, where the Si signal has low amplitude, the electronic noise of the digitizer affects the $\Delta E$ resolution of TeleD giving a worse isotopic identification. The SCT is less affected by such noise due to the higher gain of its preamplifier, thus obtaining, in spite of the reconstruction procedure, a performance similar to that of the standard telescope. The latter, however, thanks to the lower gain, features a much wider dynamic range (up to the highest $Z$ values available in the experiment, while the FEE of the SCT saturates for $Z \approx 13$ ).

At higher energy, both for hydrogen isotopes and IMF's, TeleD and SCT are quite similar in performance, giving FoM's under the 0.7 limit.

\section{Conclusions}

A $\Delta E$ - $E$ telescope exploiting the SCT concept has been tested and compared to a standard $\Delta E-E$ telescope of similar characteristics. Both are three-stage Si-Si-CsI(Tl) telescopes. In the SCT the scintillation light from the CsI(Tl) is read out by the same Si chip acting as second $\Delta E$ element. Detector signals from the preamplifiers are digitized using custom FEE and stored on disk. Information about $\Delta E$ and $E_{\mathrm{RES}}$ is extracted using a numerical reconstruction procedure based on two digital shaping filters with different time constants, acting on digitized signals.

The particle identification capability of the SCT and associated reconstruction procedure has been evaluated for different isotopes at various energies and it has been compared with that of the standard telescope. Similar performances are obtained for LCP's. For IMF's, the standard telescope gives better isotopic identification.
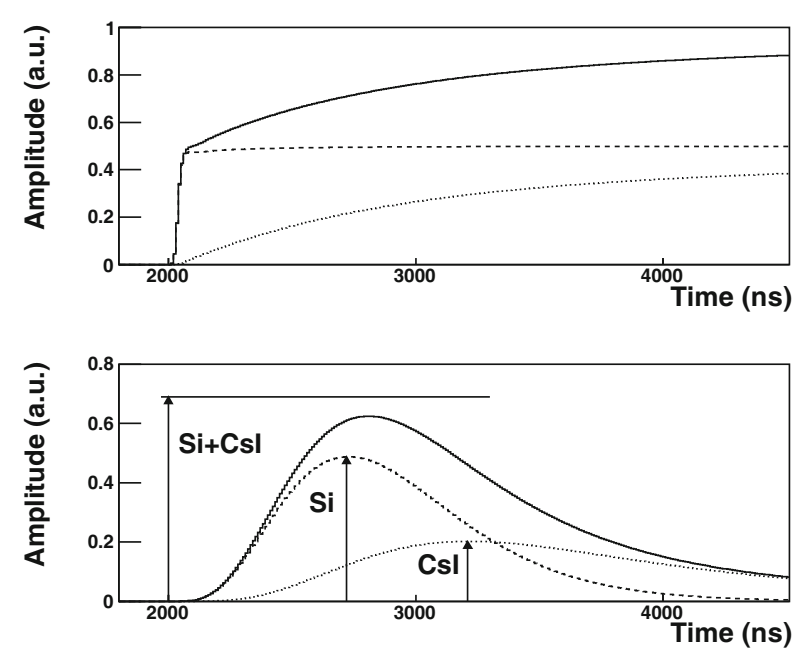

Fig. 11. Different peaking times of the short shaper for the Si and $\operatorname{CsI}(\mathrm{Tl})$ components and their effect on the overall peak value. Top panel: simulated SCT signal (solid line) for a particle punching through the Si detector and stopped in $\mathrm{CsI}(\mathrm{Tl})$. The Si (dashed line) and CsI(Tl) (dotted line) components of the signal are also separately plotted. Bottom panel: response of the short semi-Gaussian shaper to the signals shown in the upper panel. The arrow marked Si (CsI) corresponds to the maximum shaper amplitude for the $\mathrm{Si}(\mathrm{CsI})$ component. The arrow marked $\mathrm{Si}+\mathrm{CsI}$ indicates the sum of the two maximum amplitudes.

The effect of the reconstruction procedure has been studied for IMF's, analyzing SCT-like signals obtained by suitably scaling and adding the standard telescope Si and $\mathrm{CsI}(\mathrm{Tl})$ signals. It has been found that adding the signals and then applying a reconstruction procedure indeed produces worse isotopic resolution for IMF's of relatively low energy although at higher energy the standard telescope and the SCT give comparable results.

Alternative reconstruction procedures can also be devised. A procedure based on a fit of the SCT signal shapes is presently under study: its result will be published in a forthcoming paper [19].

\section{Appendix A. Correction for shaper non-linearity}

If eqs. (2) are valid, then the $Q_{I}$ and $Q_{L}$ values reflect the actual charge created in the silicon depletion volume by direct ionization and scintillation light, respectively. In particular, $\Delta E\left(\mathrm{Si}_{\mathrm{SCT}}\right)$ (given by the first of eqs. (3)), which is an estimate of $Q_{I}$, should exhibit a linear behaviour with deposited energy as expected for a standard $\mathrm{Si}$ detector. One can check this assumption by calibrating in energy the two silicon detectors and comparing the resulting $\Delta E\left(\mathrm{Si}_{1}\right)$ vs. $\Delta E\left(\mathrm{Si}_{\mathrm{SCT}}\right)$ correlation with the predictions of energy loss tables [28].

This has evidenced a discrepancy for energetic particles that punch through the second silicon detector and are stopped in the $\mathrm{CsI}(\mathrm{Tl})$. The $\Delta E\left(\mathrm{Si}_{\mathrm{SCT}}\right)$ value seems 
more and more underestimated as the total energy of the particle increases. Actually, eqs. (1) do not take into account that the maximum amplitude for the actual composite SCT signal is not equal to the sum of the maximum values one would obtain for the $\mathrm{Si}$ (direct ionization) and $\mathrm{CsI}(\mathrm{Tl})$ (scintillation) components separately. This is due to the different peaking times of the shaper when applied to the two components. The effect exists for both shapers, but it is greater for the short shaper. In fact its shaping constant is shorter than both $\mathrm{CsI}(\mathrm{Tl})$ time constants $\left(\tau_{f} \simeq 750 \mathrm{~ns}\right.$ and $\left.\tau_{s} \simeq 5 \mu \mathrm{s}\right)$ while it is greater than the collection time for charge directly produced in the silicon: therefore its peaking times for $\mathrm{Si}$ and $\mathrm{CsI}(\mathrm{Tl})$ components are the most different.

Our shapers are linear time-invariant systems. Their response to the whole SCT signal is therefore equal to the sum of their responses to the two separate components. In fig. 11 (top panel) a simulated SCT signal (solid line) is shown together with its $\mathrm{Si}$ (dashed line) and $\mathrm{CsI}(\mathrm{Tl})$ (dotted line) components. Responses of the short shaper to the full signal and separately to its two components are also shown in fig. 11 (bottom panel). Summing the peak values of the separate responses (evidenced by arrows in the bottom panel of fig. 11) one gets a value which is greater by about $10 \%$ than the response to the whole signal: in fact, when the response to the whole signal arrives at its maximum, the response to the $\mathrm{CsI}(\mathrm{Tl})$ component has not yet reached its peak amplitude. The magnitude of the effect depends on the relative weights of the silicon and $\mathrm{CsI}(\mathrm{Tl})$ components, thus producing a non-linearity in the response as a function of energy. With increasing kinetic energy, the CsI( $\mathrm{Tl})$ contribution to the SCT signal increases and this non-linear behaviour of the maximum amplitude of the shaper must be taken into account.

A correction for this effect can be estimated, based on the known shaper response. SCT signals have been simulated for different values of the $Q_{I} /\left(Q_{I}+Q_{L}\right)$ ratio. Different values of the ratio $Q_{s} / Q_{f}$ between the slow and fast $\mathrm{CsI}(\mathrm{Tl})$ components have also been used (namely 0.2 , 0.5 and 1.0). The ratio $R=\mathrm{S} / \hat{\mathrm{S}}=\mathrm{S} /\left(Q_{I}+\alpha Q_{L}\right)$ between the simulated short shaper peak amplitude and the predictions of eqs. (1) has been calculated. The $\alpha$ value corresponds to the output amplitude of the short shaper when applied to a simulated SCT signal with $Q_{I}=0$ and $Q_{L}=1$.

It is useful to study $R$ as a function of $X_{S}=Q_{I} /\left(Q_{I}+\right.$ $\left.\alpha Q_{L}\right) . X_{S}$ takes values between $X_{S}=0$, when no direct ionization in $\mathrm{Si}$ is produced $\left(Q_{I}=0\right)$, and $X_{S}=1$, when there is no contribution from $\mathrm{CsI}(\mathrm{Tl})$ scintillation light $\left(Q_{L}=0\right)$ : at the extremes of the interval spanned by $X_{S}$ one expects $R=1$. In fact, for $X_{S}=0$ and $X_{S}=1$ the full signal has only one component and thus there are no two distinct peaking times. The simulation shows that $R$ attains a minimum for $X_{S} \approx 0.5$. For instance, for the short shaper employed in this work and for $Q_{s} / Q_{f}=0.2$ (a value typical for IMF's [30]), $R$ assumes a miminum value of about 0.87 . The behaviour of $R$ as a function of $X_{S}$ is well described by a second order polynomial, whose parameters can be extracted with a fit and then used in data analysis to correct the experimental $\mathrm{S}$ values, to restore the validity of eqs. (1). We found that the maximum value of the correction to be applied to the short shaper amplitude is $13 \%$, as in the previous example, almost indipendently of the assumed $Q_{s} / Q_{f}$ ratio.

In order to apply the correction to experimental data, an estimate of $X_{S}$ is needed for each event. As a first approximation, one could assume the validity of eqs. (2), thus deriving for $X_{S}$ the following expression

$$
X_{S}=\frac{Q_{I}}{Q_{I}+\alpha Q_{L}} \simeq \frac{\mathrm{S}-K_{L} \mathrm{~L}}{\left(1-K_{L}\right) \mathrm{S}},
$$

where $\mathrm{S}$ and $\mathrm{L}$ are the experimental peak values. $K_{L}(=$ $\alpha / \beta)$ must be experimentally estimated from the $\Delta E-E$ correlations (see sect. 3.1).

A similar correction can be applied to the long shaper, though in this case the maximum correction is about $2 \%$, because of the longer peaking time.

Open Access This is an open access article distributed under the terms of the Creative Commons Attribution License (http://creativecommons.org/licenses/by/3.0), which permits unrestricted use, distribution, and reproduction in any medium, provided the original work is properly cited.

\section{References}

1. J. Pouthas et al., Nucl. Instrum. Methods A 357, 418 (1995) doi:10.1016/0168-9002(94)01543-0.

2. S. Aiello et al., Nucl. Phys. A 583, 461 (1995) doi:10.1016/ 0375-9474(94)00705-R.

3. A. Moroni et al., Nucl. Instrum. Methods A 556, 516 (2006) doi:10.1016/j.nima.2005.10.123.

4. S. Wuenschel et al., Nucl. Instrum. Methods A 604, 578 (2009) doi:10.1016/j.nima.2009.03.187.

5. D.A. Bromley, IRE Trans. Nucl. Sci. 9, 135 (1962) doi: 10.1109/TNS2.1962.4315986.

6. W.G. Gong et al., Nucl. Instrum. Methods A 268, 190 (1988) doi:10.1016/0168-9002(88)90605-5.

7. M. Pârlog et al., Nucl. Instrum. Methods A 482, 693 (2002) doi:10.1016/S0168-9002(01)01712-0.

8. E. Galichet et al., Phys. Rev. C 79, 064614 (2009) doi: 10.1103/PhysRevC.79.064614.

9. T.X. Liu et al., Phys. Rev. C 76, 034603 (2007) doi: 10.1103/PhysRevC.76.034603.

10. M. Di Toro et al., Eur. Phys. J. A 13, 155 (2002) doi: 10.1140/epja1339-28.

11. Ad.R. Raduta, F. Gulminelli, Phys. Rev. C 75, 044605 (2007) doi:10.1103/PhysRevC.75.044605.

12. FAZIA Project: http://fazia2.in2p3.fr/spip.

13. M. Mutterer et al., IEEE Trans. Nucl. Sci. 47, 756 (2000) doi:10.1109/23.856510.

14. L. Bardelli et al., Nucl. Instrum. Methods A 654, 272 (2011) doi:10.1016/j.nima.2011.06.063.

15. S. Carboni et al., Nucl. Instrum. Methods A 664, 251 (2012) doi:10.1016/j.nima.2011.10.061.

16. G. Pasquali et al., Nucl. Instrum. Methods A 301, 101 (1991) doi:10.1016/0168-9002(91)90742-9. 
17. G. Prete et al., Nucl. Instrum. Methods A 315, 109 (1992) doi:10.1016/0168-9002(92)90689-2.

18. J. Lukasik et al., EPJ Web of Conferences 31, 00032 (2012) doi:10.1051/epjconf/20123100032.

19. G. Pasquali et al., in preparation.

20. L. Bardelli et al., Nucl. Instrum. Methods A 605, 353 (2009) doi:10.1016/j.nima.2009.03.247.

21. L. Bardelli et al., Nucl. Instrum. Methods A 602, 501 (2009) doi:10.1016/j.nima.2009.01.033.

22. H. Hamrita et al., Nucl. Instrum. Methods A 531, 607 (2004) doi:10.1016/j.nima.2004.05.112.

23. L. Bardelli et al., Nucl. Instrum. Methods A 491, 244 (2002) doi:10.1016/S0168-9002(02)01273-1.

24. http://www.linear.com/.
25. G. Pasquali et al., Nucl. Instrum. Methods A 570, 126 (2007) doi:10.1016/j.nima.2006.10.008.

26. L. Bardelli et al., Nucl. Instrum. Methods A 560, 524 (2006) doi:10.1016/j.nima.2005.12.250.

27. B.W. Loo, F.S. Goulding, D. Gao, IEEE Trans. Nucl. Sci. NS-35, 114 (1988) doi:10.1109/23.12686.

28. F. Hubert et al., Atom. Data Nucl. Data Tables 46, 1 (1990) doi:10.1016/0092-640X(90)90001-Z.

29. R.A. Winyard, J.E. Lutkin, G.W. McBeth, Nucl. Instrum. Methods 95, 141 (1971) doi:10.1016/0029-554X(71) 90054-1.

30. F. Benrachi et al., Nucl. Instrum. Methods A 281, 137 (1989) doi:10.1016/0168-9002(89)91225-4. 